\title{
Analysis and Implementation of Workflow- based Supply Chain Management System
}

\author{
Yan Tu ${ }^{1}$ and Baowen Sun ${ }^{2}$ \\ 1 Information School, Central University of Finance and Economics, \\ Beijing, 100081, P.R.China,Yolanda_tu@yahoo.com.cn \\ 2 Department of Science and Research, Central University of Finance \\ and Economics, Beijing, 100081, P.R.China,sunbaowen@263.net
}

\begin{abstract}
Because of lack of abstraction at the stage of requirement analysis, the traditional Supply Chain Management systems are not suitable to dynamic reengineering when business needs change. This paper will combine Workflow Technologies with Supply Chain Management systems (WSCM). By decomposing work into the corresponding tasks and roles and monitoring the sequence of work activities according to a defined set of rules, we can boost efficiency, reduce cost, and optimize performance of business processes management of Supply Chain.
\end{abstract}

\section{Introduction}

As Internet technologies become commonplace in businesses, an emerging necessary characteristic of purchasing, logistics, and support activities is flexibility. Therefore, supply chain functions must operate in an integrated manner in order to optimize performance. However, the dynamics of the organization and the market make this challenging. In many organizations, it is likely that the sequence of work activities on business process will be changed by customers while in process, and in most cases, these change requests are difficult to manage.

In spite of the ubiquitous presence of information technology, the value that the traditional Supply Chain Management (SCM) software creates, and integration of business processes and enterprises, the challenges of implementing these applications do exist. The factors such as incompetent infrastructure, conflicting policies and standards, and changes about the sequence of work activities pose immense pressure for the organizations to move forward. The biggest challenge among them is management for the change of business processes. The issue and a potential solution we explored in this paper focus on business process management of supply chain management.

Please use the following format when citing this chapter:

Tu, Y., Sun, B., 2007, in IFIP International Federation for Information Processing, Volume 251, Integration and Innovation Orient to E-Socicty Volumel, Wang, W. (Eds), (Boston: Springer), pp. 533-543. 
In this research, we propose a workflow-based supply chain management (WCSM) for supply chain business process definition, execution, and management. To enhance adaptability for business process reengineering, we constructed our work with three main focuses. Firstly, we define a generic workflow model (i.e. four basic control flow patterns) for design a workflow modeling subsystem. Secondly, we define a set of task status and status linkage diagram. Thirdly, we descript four kinds of task firing mechanisms to perform the desired business process according to the predefined rules. The purpose of this research is to establish a workflow-based SCM system. Further, a case study in a typical business purchase process is presented to demonstrate how the WSCM system works in the domain of procurement activities to bring benefits to organizations and purchasing managers.

The remainder of this paper is organized as followings. Section 2 introduces workflow and workflow modeling technologies, which also covers the related technologies of Petri nets and CXPN (Color Extended Petri Net). Section 3 describes the design of the workflow system architecture and management mechanism to define the workflow template involving the representation of transition (task) status, workflow pattern and token operation rules, and task firing mechanisms. Section 4 uses a typical business purchase process case as an example to demonstrate WSCM at work. The last section concludes our contributions and addresses suggestions for future researches.

\section{Related Workflow Technologies}

\subsection{Workflow}

Workflow is concerned with the automation of procedures where documents, information or tasks are passed between participants according to a defined set of rules to achieve, or contribute to, an overall business goal. Workflow Management System (WfMS) is a system that completely defines, manages, and executes workflows through the execution of software whose order of execution is driven by a computer representation of the workflow logic [1]. The main function of a WfMS is to provide procedural automation of a business process, by managing of the sequence of work activities and the invoking of appropriate human or resources associated with the various activity steps.

The benefits of workflow are promoted by the Workflow Management Coalition (WfMC) as follows:

- Improved efficiency by the elimination of many unnecessary steps;

- Better process control through the standardizing of working methods and the availability of an audit trail;

- Improved customer service through greater predictability in the levels of response to customers;

- Greater flexibility of software control over processes enabling redesign when business needs change:

- Improved processes, because focusing on processes leads to their streamlining and simplification. 


\subsection{Petri Nets and Color Extended Petri Net (CXPN)}

The Petri nets have been widely used as a workflow modeling tool in a variety of ways such as the building, analysis, and simulation of business processes. A Petri net is a graphical and mathematical modeling tool. It consists of places and transitions, and describes the relations between them. As the names of these elements show, places refer to static parts of the modeled system. The graphic representation of Petri nets is a bipartite directed graph where places are drawn as circles and transitions are drawn as bars or boxes. Logical relations between transitions and places, i.e. between events and their preconditions and consequences are represented by directed arcs. In a complex system, a consequence of an event is a condition of other events. The validity of a condition in the modeled system can be represented by presence or absence of tokens in the appropriate place in the net. The number of token represents the number of resources [2].

In our research, we extend the classical Petri nets. This extension specifies that each transition has a value (color) which refers to specific status of task. For example, if task is executed and its state is changed from "suspend" to "run", the value (color) of transition will change automatically from " 8 " to " 5 ". We call it the color extended Petri nets (CXPN).

\section{Workflow-based Supply Chain Management System Framework}

\subsection{Three-tier WSCM System Architecture}

In our research, we build a WSCM for supply chain management environment as shown in Figure 1. The system architecture is defined as three-tier model for increasing of the security of data communication and the system performance [3].

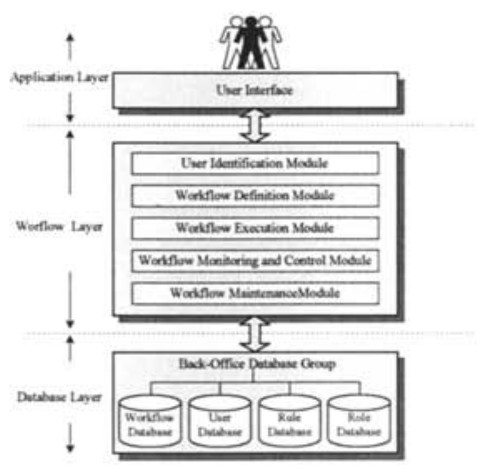

Fig. 1. Three-tier WSCM System Architecture

\subsubsection{Application Layer}

The application layer provides a graphical user interface between the user and the workflow kernel. The interface allows an authorized user to build workflow 
templates, to define workflow instance, to provide input to information forms, to interact with workflow layer, and so on.

3.1.2 Workflow Layer

The workflow layer includes a user identification module (it is used to identify user's role and the corresponding authority), a workflow definition module (it is used for the business process designer to define the workflow), a workflow execution module (its functions include interpretation of the workflow template definition, creation of workflow instances and management of their execution, including start/ready/run/ submit/suspend/resume/abort/done, etc.), a workflow monitoring and control module (its functions include supervisory and management for workflow execution), and a workflow maintenance module (it can add, delete and modify any workflow template and its relevant tasks to enhance execution performance).

3.1.3 Database Layer

The database layer provides several of back-office databases. The relevant data are stored in these databases. These data include workflow information, user information and the role model data, etc. At runtime, the system can access its associating information in these databases.

\subsection{Workflow Management Mechanism}

\subsubsection{The Representation of Transition (Task) Status}

During the execution of workflow instances, transition (referring to one task) status may change. The status linkage diagram is shown in Figure 2.

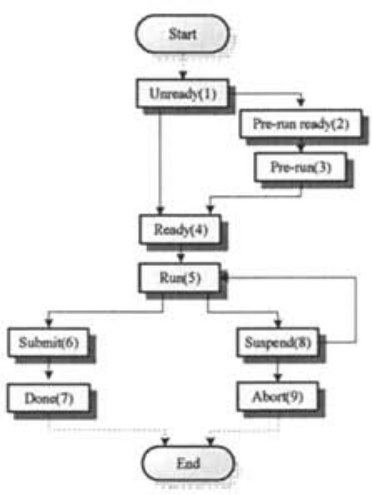

Fig. 2. Status Linkage Diagram

In CXPN, we use a finite set of colors to represent the set of transition status. The different color (different element from the color set) is corresponding with its own state. Here we use the different integer value to represent its corresponding color as shown in Table 1. 


\begin{tabular}{|c|c|c|c|}
\hline Status & No. & Status Transition & Rules Description \\
\hline Unready & 1 & $\begin{array}{l}\text { Unready-Ready } \\
\text { Unready-Pre-run ready }\end{array}$ & $\begin{array}{l}\text { All of its preconditions are fulfilled. } \\
\text { Its constraint relationship is met and pre-transition } \\
\text { is started. }\end{array}$ \\
\hline Pre-run ready & 2 & Pre-run ready-Pre-run & $\begin{array}{l}\text { Its partial pre-transitions (but not all) give the } \\
\text { results. }\end{array}$ \\
\hline Pre-run & 3 & Pre-run-Ready & $\begin{array}{l}\text { All of its pre-transitions give their own results and } \\
\text { all of its preconditions are fulfilled. }\end{array}$ \\
\hline Ready & 4 & Ready-Run & It runs according to a defined set of rules. \\
\hline Run & 5 & $\begin{array}{l}\text { Run-Submit } \\
\text { Run-Suspend }\end{array}$ & $\begin{array}{l}\text { It ends to run correctly and submits the final result } \\
\text { to system. } \\
\text { It receives the message "Suspend" or exception } \\
\text { occurs. }\end{array}$ \\
\hline Submit & 6 & Submit-Done & It stores the associated data into database. \\
\hline Done & 7 & Done-End & Workflow is end. \\
\hline Suspend & 8 & Suspend-Run & $\begin{array}{l}\text { It receives the message "Resume" or exception } \\
\text { has been handled successfully. }\end{array}$ \\
\hline Abort & 9 & $\begin{array}{l}\text { Suspend-Abort } \\
\text { Abort-End }\end{array}$ & $\begin{array}{l}\text { Exception is no way to handle. } \\
\text { Workflow is end. }\end{array}$ \\
\hline
\end{tabular}

\subsubsection{The design of the Workflow Pattern and Token Operation}

In our research, we define four basic control flow patterns: sequence pattern, parallel pattern, selection pattern and repetition pattern. We can construct the complex workflow model by using these basic patterns $[2,4]$.

To ensure the correct execution of business process, the rules of token operation should be defined accurately and the color (value) of corresponding transition (task) state should be modified dynamically in real time. Moreover, during the firing of a transition the appropriate number of tokens is removed from the input places and added to the output places. The number of added and removed tokens in a place depends on the nature of logical relations between the given place and its neighboring transitions. During the execution of workflow instances, the sequence follows the rules of token operation and the rules of task status transition that we mentioned earlier in the Table 1. The processes are illustrated by the following four basic CXPN-based workflow pattern and their associated rules.

(1) Sequence pattern. For the sequence pattern in workflow model, the associated rules to control workflow execution are that when the transition is fired and ended to execute, all of the tokens in its all of input places are removed and its all of output places add the corresponding tokens. The associated state changes from 'ready' to 'submit' and color (value) is modified from ' 4 ' to ' 6 ' as shown in Figure 3.

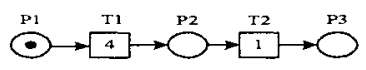

(a)

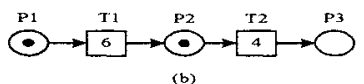

(b)

Fig. 3. Sequence pattern

(2) Parallel pattern. For the parallel pattern in workflow model, the associated rules to control workflow execution are that when the transition with AND-split is fired and ended to execute, all of the tokens in its all of input places are removed and all of output places of its corresponding transition with ANDjoin are added the tokens whose number is equal to the number of parallel 
branches. The associated state modification and the rules of token operation are the same as that of sequence pattern. It can be seen in Figure 4.

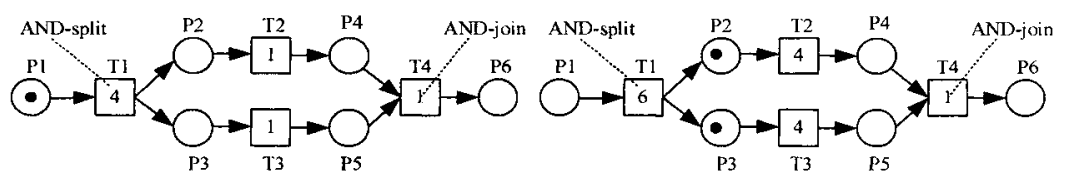

(a)

(b)

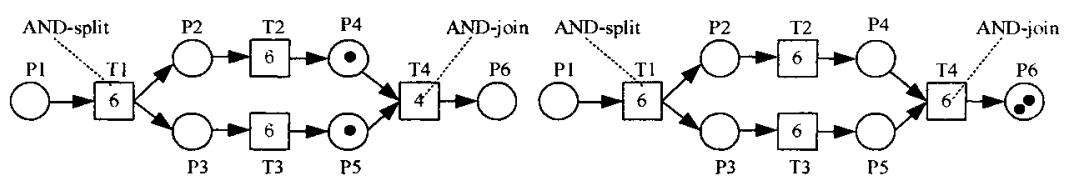

(c)

(d)

Fig. 4. Parallel pattern

(3) Selection pattern. For the selection pattern in workflow model, the associated rules to control workflow execution are that when some tokens as precondition in the place with OR-split are met for the firing condition of one or more than one of following transitions, they are fired. After they are executed, all of the tokens in places with OR-split are removed and places with OR-join are added the tokens whose number is equal to the number of token removed. The associated state modification and the rules of token operation are shown in Figure 5.

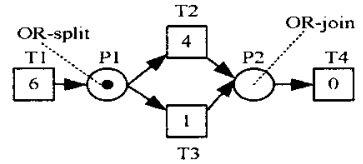

(a)

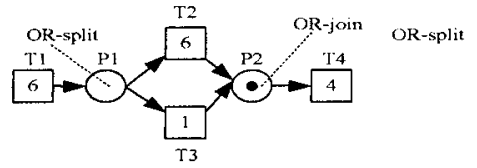

(b)

Fig. 5. Selection pattern

(4) Repetition pattern. For the repetition pattern in workflow model, its associated rules have the characteristics of both sequence pattern and selection pattern. The associated state modification and the rules of token operation are shown in Figure 6. 


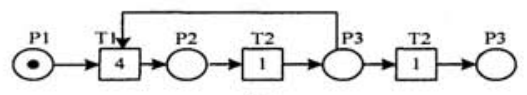

(a)

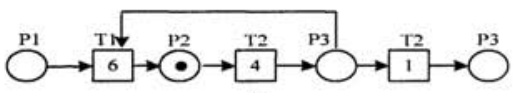

(b)

Fig. 6. Repetition pattern

\subsubsection{Firing Mechanisms of Transition}

The firing of transitions in the workflow model follows the behavior of the real life. In the model, a transition is enabled if all of its input places are valid. If a transition is enabled then it has qualification to fire. If an event occurs in the real life then the transition referring to this event must be fired in the model. Here we descript four different mechanisms about the firing of transition.

(1) Automation transitions. The firing of automation transition can occur instantaneously and automatically if all of its preconditions are fulfilled. The mark shown in Figure 7.a denotes the automation transitions.

(2) Operator intervention transitions. The firing of operator intervention transition is caused by operator interventions. Otherwise, the firing of transition can not occur without operator interventions while all of its preconditions are fulfilled. The mark shown in Figure 7.b denotes the operator intervention transitions.

(3) Message transitions. The firing of message transitions can not occur until it receives the desirable message. The mark shown in Figure 7.c denotes the message transitions.

(4) Non-primitive transitions. The firing of transition is associated with time. When the predefined time is over, the firing of transition can occur automatically. The mark shown in Figure 7.d denotes the non-primitive transitions.

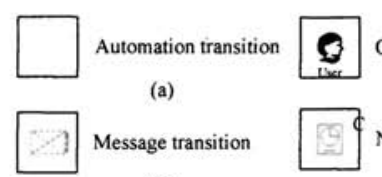

(c)
Operator intervention transition

(b)

Non-primitive transition

(d)

Fig. 7. Firing forms of transition

\section{Case Study}

In this section, we focus our works on three main parts. Firstly, process scenario as a WSCM system application is presented. Secondly, we translate it into a purchase workflow diagram. Finally, the conceptual framework is developed and the WSCM prototype is implemented for the typical business purchase processes.

\subsection{Business Purchase Process Scenario}

Business purchase is a typical case of supply chain management [5-8]. SCM project is a complementary skills working as a team for cost reductions and business process improvements. All participants contribute their expertise in different domains at various stages to reduce purchase cycle times. Purchasing 
activities include identifying vendors, evaluating vendors, selecting specific products, placing orders, and resolving any issues that arise after receiving the ordered goods or services. These issues might include late deliveries, incorrect quantities, incorrect items, and defective items. Therefore, by monitoring all relevant elements of purchase transactions, WSCM system can play an important role in maintaining and improving product quality, reducing cost, and optimizing performance of supply chain management.

\subsection{Purchase Workflow Diagram}

When a typical business purchase process is defined, we can translate it into a purchase workflow diagram, as shown in Figure 8.

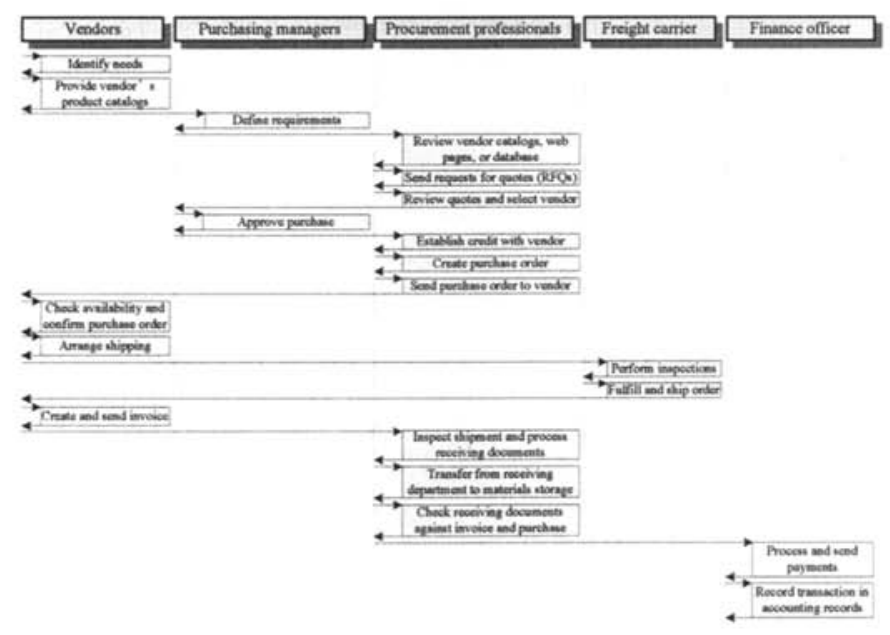

Fig. 8. Purchase workflow diagram

The diagram shows the detailed purchase workflow tasks and their corresponding team numbers. The purchase team consists of vendors, purchasing managers, procurement professionals, freight carriers, and finance officer. Based on the diagram, the workflow designer can establish a workflow template, as shown in Figure 9. 


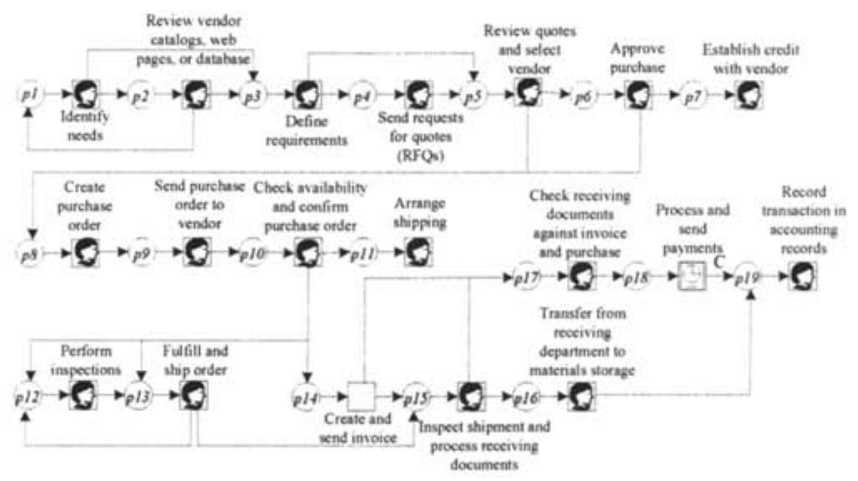

Fig. 9. Purchase workflow template

\subsection{WSCM Framework and Implementation}

Following the workflow approach, a prototype WSCM for supply chain management has been developed. Figure 10 shows the conceptual model of WSCM system. The three-tier architecture is implemented. The system includes user layer, workflow engine layer, and database layer.

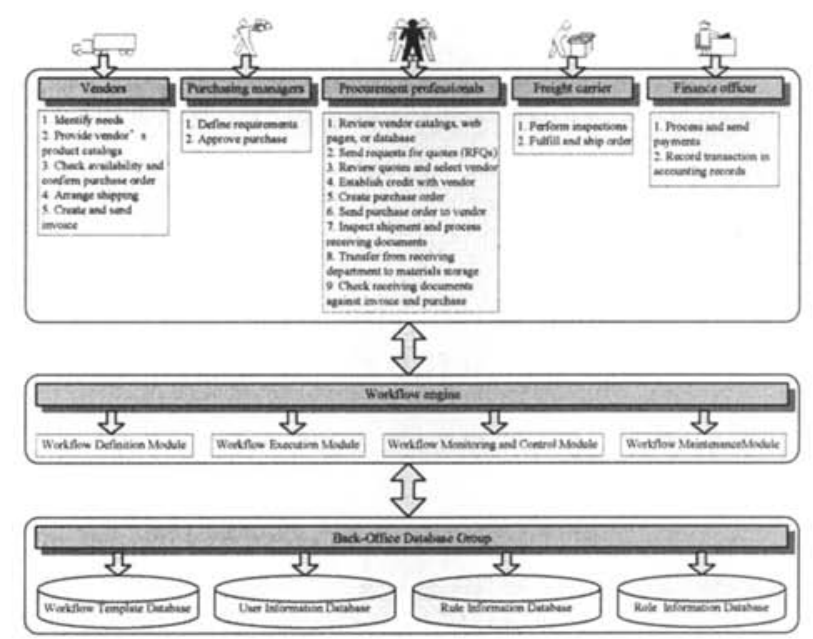

Fig. 10. WSCM Framework

Workflow designer corresponds with the process definition tool in general workflow product. In our prototype, the process definition interface is shown in Figure 11. It provides a graphic user interface by which workflow designer can model the control flow specification of workflows. Moreover, workflow designer can also assign all corresponding team members to specific tasks dynamically by dragging the elements from member's name list located at the right of the interface to workflow modeling panel. 


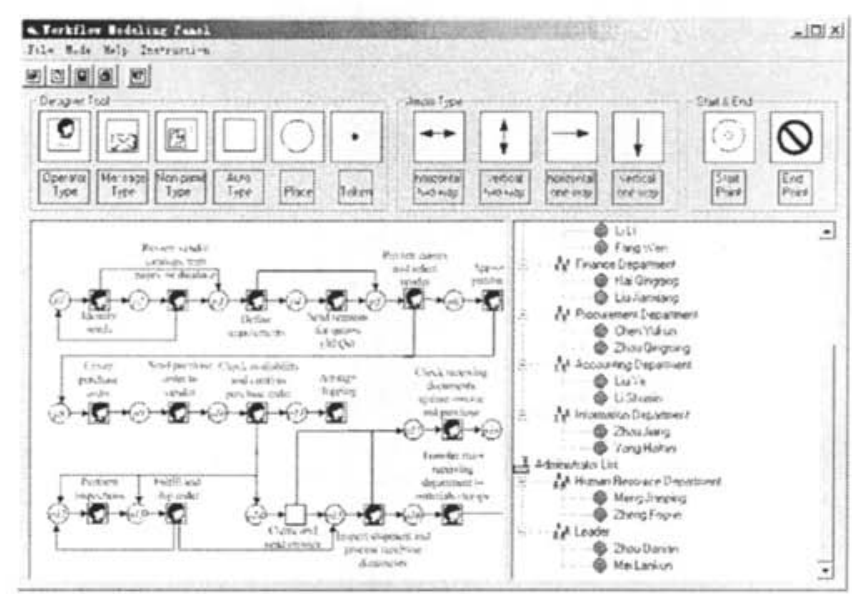

Fig. 11. Workflow Modeling Environment

After getting these information, WSCM will instantiate the workflow specification, create a workflow instance and handle its execution.

The implementation of the prototype presented in this paper is based on the four basic workflow patterns and firing mechanisms of transition. Our system mainly focuses on the implementation of the control flow perspective of workflow about supply chain management within one organization.

\section{Conclusion}

Supply chain management is the strategic, tactical, and operational level decision making that optimizes supply chain performance [5]. However, the dynamics of the organization and some exceptions can cause derivations from the business process plan. In particular, the supply chain management function is a critical link between the sources of supply and the organization. With most organizations spending at least one-third of their overall budget to purchase goods and services, the improvement of SCM performance holds significant business value. In this paper, we proposed the workflow-based supply chain management and implemented its prototype. The benefits of implementing WSCM are summarized as follows.

- It supports performance auditing and evaluation of purchase team in real time:

- Managers can optimize performance of business processes management of supply chain dynamically and flexibly;

- It supports the dynamic reengineering when business needs change;

- It provides the graphic panel with workflow designers to create and restructure workflow template.

However, our system has certain limitation. Our prototype WSCM supports supply chain management very well only within one organization. The implementation of the cross-organizational workflow product will be the focus of future research. 


\section{References}

1. Workflow Management Coalition, Workflow Management Coalition Terminology \& Glossary, Document Number WFMC-TC-1011 Document Status-Issue 3.0, 8-13(1999).

2. K.M. Hangos, Intelligent control systems, Kluwer Academic Publishers, 153-155 (2001).

3. C.J. Huang, J.C. Trappey and Y.H. Yao, "Developing an Agent-based Workflow Management System for Collaborative Product Design”, Industrial Management \& Data Systems, 106 (5), 680-699(2006).

4. H.B. Luo, Y.S. Fan, "CIMFlow: A Workflow Management System Based on Integration Platform Environment", $7^{\text {th }}$ IEEE International Conference on Emerging Technologies and Factory Automation, 233-241 ( 1999).

5. L. S. Margaret, Global Integrated Supply Chain Systems, Hershey, PA, USA: Idea Group Publishing(2005).

6. V. N. Jeffrey, "Logical Channels: Using Web Services for Cross-organizational Workflow", Business Process Management Journal,11 (3), 224-236(2005).

7. U. Mihaela, W. B. Robert and S. W. Scott, "The Holonic Enterprise: AModel for Internet-enabled Global Manufacturing Supply Chain and Workflow Management", Integrated Manufacturing systems, 538-550(2002).

8. P. S. Gary, "Electronic Commerce, Seventh Annual Edition", Thomson Learning, 217-239 (2006). 